\title{
Effects of a Free Water Protocol on Inpatients in a Neuro-Rehabilitation Setting
}

\author{
Sepideh Pooyania ${ }^{1}$, Laura Vandurme ${ }^{2}$, Reesa Daun ${ }^{2}$, Caitlin Buchel ${ }^{3}$ \\ ${ }^{1}$ University of Manitoba, Manitoba, Canada \\ ${ }^{2}$ Riverview Health Center, 1 Morley Ave, Winnipeg MB, Canada \\ ${ }^{3} 178$ Niagara Street, Winnipeg, MB, R3N 0V2, Canada \\ Email: 'spooyania@rhc.mb.ca, Ivandurme@rhc.mb.ca,rswift@rhc.mb.ca,caitlinbuchel@buchelgroup.ca
}

Received 7 September 2015; accepted 6 November 2015; published 9 November 2015

Copyright (C) 2015 by authors and Scientific Research Publishing Inc.

This work is licensed under the Creative Commons Attribution International License (CC BY).

http://creativecommons.org/licenses/by/4.0/

c) (i) Open Access

\begin{abstract}
Background: Dysphagia is common among stroke and acquired brain injury (ABI) patients and may result in aspiration. To reduce the risk of thin liquid aspiration, patients are often restricted to thickened fluids. There is considerable clinical interest in the risks and benefits of offering oral water intake, in the form of water protocols, to patients with thin-liquid dysphagia. Objectives: A controlled pilot study of a free water protocol was undertaken at Riverview Health Centre, in Winnipeg, Manitoba to assess benefits, feasibility and possible complications of free water protocol. Methods: The study examined 16 individuals with stroke or ABI who were randomly assigned to either a control group on thickened fluids (six subjects) or a treatment group (ten subjects) that followed a free water protocol. The average length of time in the study was 4.3 weeks with a range of 1 - 17 weeks among all participants. Results: Throughout the study, there was no incidence of pneumonia in any of the sixteen participants. No statistically significant difference was noted in the weekly total liquid intake between the treatment group and the control group. Perceived swallowing related care results suggest statistically significant improvements from initial to final measures for both the treatment group $(p=0.004)$ and the control group $(p=0.02)$. However, $a$ comparison of the change in both measures, between the treatment and control groups, shows no statistically significant differences. Conclusion: This pilot study suggests the need for larger scale work in order to more accurately identify the effects of free water protocols.
\end{abstract}

\section{Keywords}

Free Water Protocol, Dysphagia, Stroke and Acquired Brain Injury

\footnotetext{
"Corresponding author.
}

How to cite this paper: Pooyania, S., Vandurme, L., Daun, R. and Buchel, C. (2015) Effects of a Free Water Protocol on Inpatients in a Neuro-Rehabilitation Setting. Open Journal of Therapy and Rehabilitation, 3, 132-138. 


\section{Introduction}

Dysphagia, or difficulty swallowing, is a common sequela of stroke and acquired brain injury (ABI). Authors have estimated its incidence among stroke patients between 37\% and 78\% and between 25\% and 78\% for those with ABI [1] [2]. Dysphagia can result in a number of complications including dehydration, malnutrition, and aspiration [1] [3] [4].

Effective dysphagia management requires that speech-language pathologists (SLP) balance the risk of aspiration with a patient's nutritional needs and quality of life. In the case of thin liquid dysphagia, one of the most common management techniques is to restrict a patient's liquid intake to thickened fluids [5]. The restriction to thickened fluids, however, comes with a number of potential complications. These include possible dehydration, decreased compliance with swallowing guidelines, increased risk of aspiration pneumonia due to thickened fluid aspiration, and decreased quality of life [4] [6] [7].

A number of studies indicate that when individuals are restricted to thickened fluids, they consume less than their required daily fluid intake. This puts them at risk for dehydration [8] [9]. At the same time, the active schedules typical of patients on stroke and ABI rehabilitation units are commonly accompanied by the need for higher daily fluid intake [8]. These factors combine to put stroke and ABI patients at even greater risk of dehydration which may impact their ability to fully participate in therapies [6] [8]. In addition, this places them at risk for a variety of negative health conditions including poor wound healing, urinary tract infections, and increased confusion [8] [10]. Mc Horney et al. [11] found decreased quality of life scores for individuals consuming thickened fluids.

While thickened fluids may limit the risk of aspiration in some patients, aspiration in isolation is not a sufficient condition for the development of aspiration pneumonia [12]-[14]. Some research argues that aspirated water, neutral in $\mathrm{pH}$ and with an absence of pathogens, can be safely absorbed by the lungs [15]. This contention is indirectly supported by additional evidence, suggesting that aspiration pneumonia can result from the colonization of aspirated material by pathogens in the oral cavity and the pharynx [12] [16] [17]. Related to this, there is also evidence to show that a dedicated oral hygiene routine decreases the risk of developing aspiration pneumonia [16]-[18].

Free water protocols (FWP) have come into clinical use in an attempt to address some of the detrimental effects of thin fluid restrictions noted above (decreased quality of life, dehydration, and decreased compliance) while still maintaining the safety afforded by the provision of thickened fluids. Although specific details may vary, like the Frazier Free Water Protocol discussed by Panther [6], these protocols allow patients with documented aspiration to consume thin water under specific conditions [19].

\section{Methods}

The RHC study was a prospective, randomized, controlled pilot study conducted at Riverview Health Centre, in Winnipeg, Canada. The study took place on the centre's Neuro-rehabilitation Units (NRUs). Individuals meeting specific eligibility criteria were enrolled into the study as they were admitted to the units. Eligible participants were to have recently suffered from a stroke or ABI, be 16 years of age or older, and be admitted on thin fluid restrictions. Confirmed aspiration or a significant risk of aspiration of thin fluids was also required among potential participants. This was determined using a Videofluoroscopic Swallowing Study (VFSS) or a Fibreoptic Endoscopic Evaluation of Swallowing (FEES).

Participants were excluded from the study if they had a history of severe pulmonary complications, immune system deficiency, severe coughing to the point of discomfort when consuming thin fluids, or severe cognitive impairment that would affect their ability to follow the FWP. The study included one treatment and one control group, and individuals were assigned as they were enrolled using a random number table.

After an informed consent was obtained, the SWAL-QOL and SWAL-CARE questionnaires were administered. Designed specifically for dysphagia research, both are validated measurement tools meant to provide information related to participants' perceived quality of life and quality of care related to swallowing. The SWAL-QOL uses 44 questions, each rated on a scale of 1 - 5, to examine the impact of swallowing disorders on a variety of daily activities, including but not limited to, food selection, desire to eat, and social interactions. The SWAL-CARE uses 15 questions, rated on a scale of either 1 - 6 or 1 - 4, to examine swallowing related care as it relates to the advice received from swallowing clinicians, confidence in care, goals of treatment, and other items. 
Once these questionnaires were completed, individuals were assigned to either the treatment or control group. Participants assigned to the control group remained exclusively on thickened fluids-either honey thick or nectar thick textures. Thickened fluid intake was continuously monitored and recorded by staff, caregivers, and/or participants themselves.

Participants assigned to the treatment group continued to receive thickened fluids for meals and snacks, however they were provided with thin water between meals. A safe swallowing guide identifying the current diet texture, individual swallowing strategies, and the rules for the FWP were placed in the participant's room. These items were also placed in the shift change communication binder, and the medication administration record. As with the control group, an oral care guide listing necessary products and the required level of assistance for cleaning the patient's oral cavity was posted in their room.

As with the control group, thickened fluid intake was monitored and continuously recorded by staff, caregivers, and/or the participants themselves. However, participants were also provided with a graduated water bottle from which they were instructed to exclusively drink thin water. Instructions for measuring fluid intake from this water bottle were provided to each participant, their caregivers, and the staff at the NRU. Thin water intake among members of the treatment group was continuously recorded throughout their participation in the study.

While in the study, nursing staff monitored the participant's temperature and chest status on a daily basis. Weekly blood work was completed on each participant in order to monitor for signs of infection and/or dehydration. Participants remained in the study until they met one of a number of discharge criteria. These criteria included improvement of dysphagia resulting in an upgrade to thin fluids, participant request to withdraw from the study, the development of medical complications, or discharged from RHC. The SWAL-QOL and SWALCARE were administered to all participants a second time prior to their discharge from the study.

During the study, there were a number of issues related to the use of both of the SWAL-QOL and SWAL-CARE. Although some participants were able to independently complete both questionnaires in their entirety, some participants were not able to complete all parts as a result of significant cognitive and/or communication deficits. Administrative challenges during the data collection stage of the study also resulted in incomplete subsections on the SWAL-QOL for many of the participants. Finally, proxy reporting by friends or family members was undertaken in some instances where participants could not complete one or both of the questionnaires.

Although not all questions on the SWAL-QOL and SWAL-CARE related specifically to drinking, it was the intent of the study to consider all responses on a given questionnaire to develop overall measures of quality of life and quality of care for participants. These measures were to be developed by summing all responses and normalizing the total score to provide a rating from 0 to 1 for each participant, on each questionnaire. Here, higher scores were to indicate a higher self-assessed quality of life in the case of the SWAL-QOL and a higher quality of care in the case of the SWAL-CARE. Use of the overall measures was meant to consider quality of life across a number of dimensions simultaneously. However, as a result of the difficulties discussed above and in particular the missing subsection data on the SWAL-QOL, only the aggregate measure for the SWAL-CARE was developed.

Although five outcomes were to be measured during the study, the inability to construct an aggregate measure of swallowing related quality of life meant that four measures were examined during the analysis. The first was the occurrence of aspiration pneumonia among the participants, at any point during their participation in the study. Diagnosis of aspiration pneumonia was based on chest X-ray and physician documentation. The second and third outcomes were the total amount of weekly fluid intake and total amount of thickened fluid intake among participants. Depending on the participants' cognition, thin and thick fluid intake was either self-reported, or recorded by staff or the patients' significant others. The fourth outcome involved the 0 to 1 quality of care related measures developed from the SWAL-CARE.

From among those identified as eligible to participate, seventeen participants were enrolled between August 2010 and December 2012. This group included 16 males and one female. Participants ranged in age from 16 - 74 years old with an average age of 56.4. Prior to beginning their participation in the study, one patient was upgraded to thin fluid intake and no longer met the eligibility criteria. They were removed and no data on this individual was subsequently collected. Six participants were assigned to the control group and 10 participants were assigned to the treatment group. The average length of time in the study was 4.3 weeks with a range of 1 17 weeks among all participants. 


\section{Statistical Analysis}

The descriptive statistics presented below include means and standard deviations for four of the outcome measures examined in the study, as measured at intake or week one. Testing for group differences in these continuous, baseline or week one measures was completed using a two-tailed t-test. A series of generalized estimating equations (GEE) were used to examine whether average fluid intake between week one and discharge differed between the treatment and control groups. Finally, a series of mixed model analyses were conducted to examine changes, between intake and discharge, in swallowing related quality of care within and between groups. Use of the GEE and mixed modeling approaches allowed the study to incorporate data from all study participants, including those with missing fluid intake measurements in a given week. All reported p-values were two-sided, and significance was set at a value of 0.05. All statistical analyses were performed using SAS Version 9.3.

\section{Results}

Throughout the study, there was no incidence of pneumonia or other respiratory complications in any of the sixteen participants. Information obtained from infection control at RHC further indicated that the quarterly case rate of pneumonia for the NRUs for 2012 was 1.112 per 1000 patient days. In addition, no participant was diagnosed as being dehydrated during their participation in the study.

Table 1 presents descriptive statistics, including means and standard deviations, for three of the study's four outcomes. These include thickened and total fluid intake, as measured during participants' first week in the study. They also include swallowing related care values as measured upon enrollment into the study. Swallowing related quality of life results are not presented, due to a high number of participants failing to complete all sub-scales on the SWAL-QOL. From Table 1, we find that there is no significant difference between treatment and control groups for the three outcome measures.

As noted in Table 2, no statistically significant difference was noted in the weekly total fluid intake between the treatment group and the control group. There was also no statistically significant difference between the amounts of thickened fluids consumed on a weekly basis between groups.

Table 3 compares changes in swallowing related quality of care between initial and final measures for the control and treatment groups. It further compares these within group changes in outcomes between the treatment and control groups. Perceived swallowing related care results suggest statistically significant improvements from initial to final measures for both the treatment group $(p=0.004)$ and the control group $(p=0.02)$. However, a comparison of the change in both measures, between the treatment and control groups, show no statistically significant differences $(\mathrm{p}=0.12$ and $\mathrm{p}=0.99)$.

\section{Discussion}

There are currently three published prospective FWP studies in the literature. Garon, Engle, and Ormiston [20] completed a small randomized controlled trial $(n=20)$ in a rehabilitation setting. Much like the chart review

Table 1. Comparison of outcome measures across groups.

\begin{tabular}{cccc}
\hline Outcome & Treatment $(\mathrm{n}=10)$ & Control $(\mathrm{n}=6)$ & $\mathrm{p}$ value \\
\hline Weekly thickened liquids intake ${ }^{1}(\mathrm{~mL})$ & $2674.6 \pm 1744.0$ & $3218.7 \pm 2593.1$ & 0.65 \\
${\text { Weekly total liquids intake }{ }^{1}(\mathrm{~mL})}^{\text {Swallowing- related quality of life }}{ }^{2}$ & $2953.2 \pm 1924.9$ & $3385.33 \pm 2408.9$ & 0.70 \\
Swallowing- related care & & $\#$ & $\#$ \\
\hline
\end{tabular}

Note: Results expressed a mean \pm standard deviation. ${ }^{1}$ As measured in week one. ${ }^{2}$ As measured at intake. ${ }^{\#}$ Results omitted due to incomplete SWAL-QOL questionnaires.

Table 2. Comparison of average weekly liquid intake.

\begin{tabular}{cccrcr}
\hline Outcome & Treatment & Control & Difference & p value \\
\hline Thickened liquids intake $(\mathrm{mL})$ & $3591.7 \pm 555.0$ & $4380.3 \pm 906.6$ & $-788.6(1063.0)$ \\
Total liquids intake $(\mathrm{mL})$ & $4035.3 \pm 710.8$ & $4481.4 \pm 838.3$ & $-446.1(1099.1)$ & 0.68 & \\
\hline
\end{tabular}

Note: Results expressed a mean \pm standard error. Liquid intake measured between week 1 and week 7. 
Table 3. Changes in quality of life outcome measures.

\begin{tabular}{|c|c|c|c|c|c|c|c|c|c|c|}
\hline \multirow[b]{2}{*}{ Outcome } & \multicolumn{4}{|c|}{ Treatment } & \multicolumn{4}{|c|}{ Control } & \multicolumn{2}{|c|}{$\begin{array}{l}\text { Treatment vs. } \\
\text { Control }\end{array}$} \\
\hline & Base-line & Dis-charge & $\begin{array}{l}\text { Change from } \\
\text { pre to discharge } \\
(95 \% \mathrm{CI})\end{array}$ & $\mathrm{p}$ & Base-line & Dis-charge & $\begin{array}{l}\text { Change from } \\
\text { pre to discharge } \\
(95 \% \mathrm{CI})\end{array}$ & $\mathrm{p}$ & $\begin{array}{l}\text { Estimate } \\
(95 \% \text { CI })\end{array}$ & $p$ \\
\hline $\begin{array}{l}\text { Swallowing- } \\
\text { related } \\
\text { quality of life }\end{array}$ & $\#$ & $\#$ & $\#$ & $\#$ & $\#$ & $\#$ & $\#$ & $\#$ & $\#$ & $\#$ \\
\hline $\begin{array}{l}\text { Swallowing- } \\
\text { related care }\end{array}$ & $0.49 \pm 0.15$ & $0.68 \pm 0.15$ & $0.19(0.07$ to 0.30$)$ & 0.004 & $0.59 \pm 0.14$ & $0.78 \pm 0.13$ & 0.19 (0.04 to 0.34$)$ & 0.02 & $\begin{array}{c}-0.01 \\
(-0.19 \text { to } 0.18)\end{array}$ & 0.99 \\
\hline
\end{tabular}

Note: Results expressed a mean \pm standard deviation. "Results omitted due to incomplete SWAL-QOL questionnaires.

discussed above, the experimental study identified no difference in rates of aspiration pneumonia between patients on a FWP and a control group. In addition, as in the retrospective work of Panther discussed above, the experimental group in this case also consumed greater fluids when compared to the control group.

A second controlled prospective study by Karagiannis, Chivers, and Karagiannis [21] examined 100 sub-acute patients with dysphagia resulting from a variety of medical conditions. When comparing the treatment and control groups, the authors found that total fluid intake was higher among those in the treatment group on the FWP. Yet the authors also found that $14.3 \%$ of treated patients developed lung complications while none of those in the study's control group did. While this result appears qualitatively different from those of the studies discussed above, it is worth noting that all of the patients that developed lung complications had either a neuro-degenerative disease, poor mobility, or both.

The most recently published prospective results come from a randomized controlled trial of a FWP come from the GF Strong Rehabilitation Centre in Vancouver, Canada. The results from this study appear to align with many of those previously mentioned. In their study, Carlaw et al. [22] note no adverse events from the use of a FWP with individuals in their trial. In addition, they identified increased fluid intake and reported high quality of life outcome measures among FWP participants.

Although the RHC study noted no adverse events among the treatment group, unlike in Carlaw's study, it was unable to show a statistically significant difference in either thickened fluid or total fluid intake between individuals on a FWP and a control group. Part of this inability may have resulted from the small patient sample available for the study as well as possible inconsistencies in measuring fluid intake. This may point to the need for collaborative multi-site research in order to establish whether this clinical benefit exists.

While not designed as a replication of the Carlaw study, the RHC study did have a number of features in common with this earlier work. The studies did differ, however, in two ways. First, in the Carlaw study, at least some participants were explicitly offered thick fluids throughout the trial. In the case of the RHC study, this same encouragement was not provided. Second, the Carlaw study tracked participants' fluid intake for 14 days regardless of the length of time the participant was enrolled in the study. The RHC study tracked participants' fluid intake for the entire time they were enrolled in the study. These differences may be reasons why the Carlaw study observed statistically significant changes in fluid intake while the RHC study did not. It may further reinforce the need to encourage fluid intake if FWPs are to increase fluid intake among patient populations.

Interestingly, the study was also able to point to statistically significant improvements in measures of perceived swallowing related care. This was true, however, for both groups, with no difference in these changes between groups being identified. This may suggest that both dysphagia related information and the information provided on the FWP were both well received by patients and improved their perception of their care.

With these results in mind, it is important to note that the study suffered from a number of limitations. In particular, it was not feasible to have a small group of trained staff measure and record all fluid intake by participants. This resulted in measurement by a large and diverse set of individuals including allied healthcare professionals, nursing staff, health care aides, caregivers, and participants. This also made it more difficult to establish participant compliance during the study. Overall, it is almost certain that this resulted in measurement inconsistency, the effects of which are not well understood in the RHC study.

In addition, quality of life and care measurements were complicated by the nature of the participant group, its size, and the failure of many participants to fully complete the SWAL-QOL questionnaire. Some of the individuals 
participating in the RHC study suffered from aphasia or other cognitive impairments, making it difficult if not impossible for them to effectively complete the SWAL-QOL and the SWAL-CARE. In addition, given the small number of participants it was not possible to effectively control for diet texture in the experimental design. This type of control is important given that the SWAL-QOL and the SWAL-CARE measure general aspects of swallowing that are not specifically related to fluid intake alone.

\section{Conclusion}

As suggested above, future research should attempt to enrol a larger participant sample in order to more accurately measure the effects of FWPs. There may be greater value in undertaking such work across a number of sites rather than attempting to pursue single facility research. At the same time, challenges including those mentioned above will need to be addressed while ensuring effective control of other potential factors possibly influencing the measured clinical outcomes—in particular the exact nature of the FWP implemented.

\section{Funding}

This study was supported by a research grant by Riverview Health Center Foundation and was conducted in Riverview Health Center, Winnipeg, MB, Canada.

\section{References}

[1] Martino, R., Foley, N., Bhogal, S., Diamant, N., Speechley, M. and Teasell, R. (2005) Dysphagia after Stroke: Incidence, Diagnosis, and Pulmonary Complications. Stroke, 36, 2756-2763. http://dx.doi.org/10.1161/01.STR.0000190056.76543.eb

[2] Welch-West, P., Aubut, J.-A., Foley, N. and Teasell, R. (2012) Module 5-Stroke Patients and Aspiration Pneumonia. In: Evidence-Based Review of Moderate to Severe Acquired Brain Injury, ERABI, Parkwood Hospital London, Canada.

[3] Holas, M.A., DePippo, K.L. and Reding, M.J. (1994) Aspiration and Relative Risk of Medical Complications Following Stroke. Archives of Neurology, 51, 1051. http://dx.doi.org/10.1001/archneur.1994.00540220099020

[4] Scharver, C.H., Hammond, C.S. and Goldstein, L. (2009) Post-Stroke Malnutrition and Dysphagia. In: Bales, C.W. and Ritchie, C.S., Eds., Handbook of Clinical Nutrition and Aging, 2nd Edition, Humana Press, Totowa, NJ. http://dx.doi.org/10.1007/978-1-60327-385-5_24

[5] Garcia, J.M., Chambers, E. and Molander, M. (2005) Thickened Liquids: Practice Patterns of Speech-Language Pathologists. American Journal of Speech-Language Pathology, 14, 4. http://dx.doi.org/10.1044/1058-0360(2005/003)

[6] Panther, K. (2005) The Frazier Free Water Protocol. Perspectives on Swallowing and Swallowing Disorders. Dysphagia, 14, 4-9. http://dx.doi.org/10.1044/sasd14.1.4

[7] Robbins, J., Gensler, G., Hind, J., Logemann, J.A., Lindblad, A.S., Brand, D. and Lundy, D. (2008) Comparison of Two Interventions for Liquid Aspiration on Pneumonia Incidence: A Randomized Trial. Annals of Internal Medicine, 148, 509. http://dx.doi.org/10.7326/0003-4819-148-7-200804010-00007

[8] Finestone, H.M., Foley, N.C., Woodbury, M.G. and Green-Finestone, L. (2001) Quantifying Fluid Intake in Dysphagic Stroke Patients: A Preliminary Comparison of Oral and Non-Oral Strategies. Archives of Physical Medicine and Rehabilitation, 82, 1744-1746. http://dx.doi.org/10.1053/apmr.2001.27379

[9] Whelan, K. (2001) Inadequate Fluid Intake in Dysphagic Acute Stroke. Clinical Indicators of Dehydration Severity in Elderly Patients. Journal of Emergency Medicine, 3, 267-274.

[10] Copeman, J. (2000) Promoting Nutrition in Older People in Nursing and Residential Homes. British Journal of Community Nursing, 5, 277-284. http://dx.doi.org/10.12968/bjcn.2000.5.6.7396

[11] McHorney, C.A., Robbins, J., Lomax, K., Rosenbek, J.C., Chignell, K., Kramer, A.E. and Earl Bricker, D. (2002) The SWAL-QOL and SWAL-CARE Outcomes Tool for Oropharyngeal Dysphagia in Adults: III. Documentation of Reliability and Validity. Dysphagia, 17, 97-114. http://dx.doi.org/10.1007/s00455-001-0109-1

[12] Langmore, S.E., Terpenning, M.S., Schork, A., Chen, Y., Murray, J.T., Lopatin, D. and Loesche, W.J. (1998) Predictors of Aspiration Pneumonia: How Important Is Dysphagia? Dysphagia, 13, 69-81. http://dx.doi.org/10.1007/PL00009559

[13] Feinberg, M. J., Kneble, J. and Tully, J. (1996) Prandial Aspiration and Pneumonia in an Elderly Population Followed over 3 Years. Dysphagia, 11, 104-109. http://dx.doi.org/10.1007/BF00417899

[14] Gosney, M. (2005) Stroke Patients and Aspiration Pneumonia. Perspectives on Swallowing and Swallowing Disorders (Dysphagia), 14, 16-20. http://dx.doi.org/10.1044/sasd14.1.16 
[15] Effros, R.M., Jacobs, E.R., Schapira, R.M. and Biller, J. (2000) Response of the Lungs to Aspiration. The American Journal of Medicine, 108, 15-19. http://dx.doi.org/10.1016/S0002-9343(99)00290-9

[16] Azarpazhooh, A. and Leake, J.L. (2006) Systematic Review of the Association between Respiratory Diseases and Oral Health. Journal of Periodontology, 77, 1465-1482. http://dx.doi.org/10.1902/jop.2006.060010

[17] Yoneyama, T., Yoshida, M., Ohrui, T., Mukaiyama, H., Okamoto, H., Hoshiba, K. and Morita, T. (2002) Oral Care Reduces Pneumonia in Older Patients in Nursing Homes. Journal of the American Geriatrics Society, 50, 430-433. http://dx.doi.org/10.1046/j.1532-5415.2002.50106.x

[18] Terpenning, M. (2005) Prevention of Aspiration Pneumonia in Nursing Home Patients. Clinical Infectious Diseases, 40, 7-8. http://dx.doi.org/10.1086/426030

[19] Frey, K.L. and Ramsberger, G. (2011) Comparison of Outcomes before and after Implementation of a Water Protocol for Patients with Cerebrovascular Accident and Dysphagia. The Journal of Neuroscience Nursing: Journal of the American Association of Neuroscience Nurses, 43, 165-171. http://dx.doi.org/10.1097/JNN.0b013e3182135adf

[20] Garon, B.R., Engle, M. and Ormiston, C. (1997) A Randomized Control Study to Determine the Effects of Unlimited Oral Intake of Water. Journal of Neurologic Rehabilitation, 11, 139-148. http://dx.doi.org/10.1177/154596839701100301

[21] Karagiannis, M.J.P., Chivers, L. and Karagiannis, T.C. (2011) Effects of Oral Intake of Water in Patients with Oropharyngeal Dysphagia. BMC Geriatrics, 11, 9. http://dx.doi.org/10.1186/1471-2318-11-9

[22] Carlaw, C., Finlayson, H., Beggs, K., Visser, T., Marcoux, C., Coney, D. and Steele, C.M. (2011) Outcomes of a Pilot Water Protocol Project in a Rehabilitation Setting. Dysphagia, 27, 297-306.

http://dx.doi.org/10.1007/s00455-011-9366-9 